KYUNGPOOK Math. J. 51(2011), 311-322

http://dx.doi.org/10.5666/KMJ.2011.51.3.311

\title{
Some Properties of the Closure Operator of a Pi-space
}

HuA MAO*

Department of Mathematics, Hebei University, Baoding 071002, China

e-mail : yushengmao@263.net

SANYANG LiU

Department of Mathematics, Xidian University, Xi'an 710071, China

e-mail : liusanyang@126.com

Abstract. In this paper, we generalize the definition of a closure operator for a finite matroid to a pi-space and obtain the corresponding closure axioms. Then we discuss some properties of pi-spaces using the closure axioms and prove the non-existence for the dual of a pi-space. We also present some results on the automorphism group of a pi-space.

\section{Introduction and preliminaries}

With the development of matroid theory, we have new tools to consider infinite matroid-like structures. Also, studying on these infinite structures will accelerate research in matroid theory. But we should notice that Oxley [17] pointed out: "There is no single class of structures that one calls infinite matroids. Rather, various authors with differing motivations have studied a variety of classes of matroid-like structures on infinite sets."

We may observe that recently, most of results on infinite matroids are relative to pi-spaces, independence spaces and matroids of arbitrary cardinality (cf. [1, $6-15,17,20]$ and the references in $[1,6-15,17,20])$. On the other hand, the definition of an independence space (cf. [17\&20, p. 387]) directly indicates that an independence space is a pi-space. Additionally, from the concept of a matroid of arbitrary cardinality (cf. [1]) and the discussion in [20, Chapter 20], we can know that a matroid of arbitrary cardinality is an independence space, and so, a pi-space. In one word, a class of structures in infinite matroids which has been most fruitfully used seems to be pi-spaces. We may equivalently state that pi-spaces are one of the more frequently studied classes of infinite matroids.

$[18,20,21]$ show that closure operators of finite matroids are a bridge between finite matroids and geometries. [7] obtains the relation between geometric lattices

\footnotetext{
* Corresponding Author.

Received April 16, 2010; accepted October 28, 2010.

2010 Mathematics Subject Classification: 05B35.

Key words and phrases: pi-space, closure operator, closed set, dual, automorphism.
} 
and simple matroids of arbitrary cardinality with the help of the closure operators of matroids of arbitrary cardinality. Using this relation, [8-10,12,14,15] deal with some properties of matroids of arbitrary cardinality. These results represent that it is important to notice the properties of closure operators of matroids of arbitrary cardinality. Similarly to the discussion for finite matroids and matroids of arbitrary cardinality, we should better find out the relationship between pi-spaces and closure operators. Unfortunately, to our knowledge, few authors have searched out the relationships between pi-spaces and closure operators. Hence, to study on pi-spaces more detailed, the first step is to solve the closure axiom systems for pi-spaces. The second is to deal with the properties of the closure operator for a pi-space.

The purpose of this paper is to generalize the notation of closure operator from finite matroids to pi-spaces and find out the closure axiom systems for a pi-space. Simultaneously, it deals with the non-existence of the dual of a pi-space by the hand of a successful example. At the last part, for a pi-space, it discusses some properties of its automorphism group under the help of closure operator.

Initially, we will recall and present some basic knowledge. In the following, we will work over a ground-possibly infinite-set $S$; the set of all subsets of $S$ will be denoted by $2^{S} ; X \subset \subset Y$ indicates that $X$ is a finite subset of $Y$. A set system over $S$ is a non-empty family contained in $2^{S}$. Let $\mathcal{A} \subseteq 2^{S}$ be a set system. We say that a basis of a subset $A \subseteq S$ in $\mathcal{A}$ is a (inclusion-wise) maximal element of $A$ in $\mathcal{A}$.

Throughout this paper, all the knowledge of lattice theory are referred to [2] and [3]; group theory is come from [4]; finite matroid theory is seen [18], [20] and $[21]$.

Definition 1. (1)[17\&20] A pi-space $M$ is a set $S$ together with a collection J of subsets of $S$ (called independent sets) such that

(i1) $\mathcal{J} \neq \emptyset$.

(i2) If $A \in \mathcal{J}$ and $B \subseteq A$, then $B \in \mathcal{J}$.

(i3) If $A, B$ are finite members of $\mathcal{J}$ with $|A|=|B|+1$, then there exists $a \in A \backslash B$ such that $B \cup a \in \mathcal{J}$.

(2) [20, p.386] With a pi-space $(S, \mathcal{J})$, we associate a rank function taking values from $\{0,1,2, \ldots, \infty\}$ and defined for $X \subseteq S$ by $\rho(X)=\sup \{|Y|: Y \in \mathcal{J}, Y \subset \subset X\}$.

As finite matroids, we define the closure operator of $M=(S, \mathcal{J})$ as follows.

Definition 2. A function $\sigma: 2^{S} \rightarrow 2^{S}$ is the closure operator of pi-space $M$ if $\sigma(X)=\{y \in S: \rho(X \cup y)=\rho(X)\} . X$ is called a closed set of $M$ if $\sigma(X)=X$.

In the following, $M=(S, \mathcal{J})$ denotes a pi-space with $\sigma, \rho$ as its closure operator and rank function respectively. By Definition 2, we see that for a pi-space $M$, the construction of $\mathcal{F}$ of set of closed sets of $M$ is seemingly simpler than that of $\mathcal{J}$. Actually, $\mathcal{F}=\{X: \sigma(X)=X\}$. Hence, studying on $\mathcal{F}$ is perhaps better to the research on some properties of $M$. In addition, we may easily know $\sigma(A)=A \cup\{y \in$ $S \backslash A: \rho(A \cup y)=\rho(A)\}$ for $A \subseteq S$. Therefore, in Section 2, we mainly study on the closure axioms with assistance of $\rho$ and $\mathcal{J}$. 
Some people will think that this literature is not new because of $[5,19]$. But the following reasons and analysis will indicate that the paper is valuable to be read.

(RI) [5] is one of the most important references of [19] (cf. [19, p. 348 etc.]) and most of results of [5] relative to matroids are contained in [19]. Hence, we pay attention to [19].

(RII) [20] informs us that MacLane's condition is important to finite matroids, especially for closure operators of matroids. Pi-spaces are the generalization of finite matroids. Hence, when we consider pi-spaces, MacLane's condition is in the consideration. In other words, we should pay our attention to closure operators of pi-spaces.

(RIII) Using lattice theory, for finite and infinite cases, [19] has discussed some results about semimodular lattices, in particular, MacLane's condition on semimodular lattices. But it does not use matroid theory to their discussion, and in addition, it does not discuss the relation between matroids and semimodular lattices. Of course, it does not touch upon the closure axioms of infinite matroids, especially pi-spaces.

(RIV) [20] points out:

$(\alpha)$ There are correspondent relationships between finite matroids and finite geometric lattices. In addition, for a finite geometric lattice, we may find a unique simple matroid to correspond with this geometric lattice under isomorphism.

$(\gamma)$ For non-simple matroids, the above corresponding will not be true.

$(\alpha)$ and $(\gamma)$ demonstrate that lattice theory will not replace matroid theory.

(RV) [20] indicates that it is not easy to translate the language from matroids to geometric lattices, and vice versa, though this is essential to the study between matroids and lattices. [19] does not realize the translation of language from lattice theory to matroid theory.

The two views together verifies that the results in [19] can not replace the discussion to pi-spaces applying matroid theory language.

(RVI) Using the language of matroid theory, this paper finds out the closure axioms for a pi-space, some properties of pi-spaces on lattice theory and some other results. All the discussion in this paper are finished under the notions of matroid theory, not lattice theory. Hence, we may assert that our consequences are different from $[5,19]$.

\section{Closure axioms}

$[18,20,21]$ demonstrate the importance of closure operators in finite matroids. Here we examine more elementary ideas and axioms for pi-spaces with their closure operators.

Let $A, B \subseteq S$ and $x, y \in S$. Then there are the following Lemma 1 and Lemma 2 .

Lemma 1. (1) $\rho(A) \leq \rho(B)$ if $A \subseteq B \subseteq S$. 
(2) When $\rho(A)<\infty$. There is $I_{A} \subset \subset A$ with $I_{A} \in \mathcal{J}$ satisfying $\left|I_{A}\right|=\rho(A)$. In addition, all maximal elements of $A$ in $\mathcal{J}$ have the same values.

(3) When $\rho(A)=\infty$. It follows $\sigma(A)=S$.

(4) If $A \subseteq S$ and $x \in S$, then $\rho(A)=\rho(\sigma(A))$ and $\rho(A \cup x)=\rho(\sigma(A) \cup x)$.

Proof. (1) is straightforward from Definition 1.

(2) is testified by Definition 1 and (i2).

(3) is evident by Definition 2.

(4) will be proved as follows.

First, we prove $\rho(A)=\rho(\sigma(A))$.

By (1), it follows $\rho(A) \leq \rho(\sigma(A))$. Suppose $\rho(A)<\rho(\sigma(A))$. Then $A \subset \sigma(A)$ is true. Divided two cases for discussion.

Case 1. $\rho(\sigma(A))<\infty$.

According to (2), there is $I \subset \subset \sigma(A)$ and $I \in \mathcal{J}$ satisfying $\rho(\sigma(A))=|I|$. In view of $A \subset \sigma(A)$ and $\rho(A)<\rho(\sigma(A))$, it induces $I \cap\{y \in S \backslash A: \rho(A \cup y)=$ $\rho(A)\}=I_{S \backslash A} \neq \emptyset$. However, since $\left|I_{S \backslash A}\right|<\infty$ is correct owing to $\left|I_{S \backslash A}\right| \leq|I|<\infty$. Additionally, $\rho(A \cup y)=\rho(A)$ holds for any $y \in I_{S \backslash A}$ because of $I_{S \backslash A} \subseteq I \subseteq \sigma(A)$. These follow $\rho\left(A \cup I_{S \backslash A}\right)=\rho(A)$. Let $I_{A}=I \cap A . \rho\left(A \cup I_{A}\right)=\rho(A)$ is evident in virtue of $I_{A} \subseteq \sigma(A)$ and Definition 2 .

Therefore, $\rho(A \cup I)=\rho\left(A \cup I_{A} \cup I_{S \backslash A}\right)=\rho(A)$ is real. Further, $|I| \leq \rho(A \cup$ $I)=\rho(A)$ holds. So $\rho(\sigma(A)) \leq \rho(A)$ is born. This leads to a contradiction to $\rho(A)<\rho(\sigma(A))$.

Case 2. $\rho(\sigma(A))=\infty$.

In light of $\rho(A)<\rho(\sigma(A))$, it carries out $\rho(A)<\infty$. For any $X \in\{Y: Y \subset \subset$ $\sigma(A), Y \in \mathcal{J}\}$, analogous to the proof in Case 1, we obtain $\rho(A \cup X)=\rho(A)$, and so, $|X| \leq \rho(A \cup X)=\rho(A)<\infty$. Thus, it has $\rho(\sigma(A))=\sup \{|Y|: Y \subset \subset \sigma(A), Y \in$ $\mathcal{J}\} \leq \rho(A)$, a contradiction.

Summing up Case 1 and Case 2, it follows $\rho(A)=\rho(\sigma(A))$.

Second, let $x \in S$, we prove $\rho(A \cup x)=\rho(\sigma(A) \cup x)$.

Owing to (1), it gets $\rho(A) \leq \rho(A \cup x) \leq \rho(\sigma(A) \cup x)$.

Assume $\rho(A)=\infty$. Then it has $\rho(A \cup x)=\rho(\sigma(A) \cup x))=\infty$.

Assume $\rho(A)<\infty$. According to $(2)$, there is $I_{A} \in \mathcal{J}$ and $I_{A} \subseteq A$ satisfying $\left|I_{A}\right|=\rho(A)$. This also shows that $I_{A}$ is a maximal of $A$ in J. Additionally, every maximal of $A$ in $\mathcal{J}$ is also a maximal of $\sigma(A)$ in $\mathcal{J}$ because of $\rho(A)=\rho(\sigma(A)$ ), $A \subseteq \sigma(A)$ and Definition 2. Thus, considering with $\rho(A)<\infty$, Definition 1 and (2), we obtain $\rho(A \cup x) \leq \rho(A)+1<\infty$. If $I \cup x \in \mathcal{J}$ with $I \cap\{x\}=\emptyset$ and $I \subseteq A$ satisfies $\rho(A \cup x)=|I \cup x|=|I|+1=\rho(A)+1$, then we may see that $I$ fits to $\rho(A)=|I|$. Furthermore, $I \cup x$ satisfies $\rho(\sigma(A) \cup x)=\rho(I \cup x)=|I \cup x|=|I|+1=\rho(\sigma(A))+1$. Thus, $\rho(A \cup x)=\rho(\sigma(A) \cup x)$ is gotten.

Lemma 2. $\sigma$ satisfies $(s 1)-(s 5)$.

(s1) $A \subseteq \sigma(A)$.

(s2) $A \subseteq B \Rightarrow \sigma(A) \subseteq \sigma(B)$.

(s3) $\sigma(\bar{A})=\sigma(\sigma(A))$.

(s4) $y \notin \sigma(A)$ and $y \in \sigma(A \cup x) \Rightarrow x \in \sigma(A \cup y)$. 
(s5) If $Y_{1} \subset Y_{2} \subset \ldots \subset Y_{j} \subset Y_{j+1} \subset \ldots \subset X,\left|Y_{j}\right|=j, Y_{j+1} \backslash Y_{j} \neq \emptyset,(j=$ $1,2, \ldots), \sigma(\emptyset) \subset \sigma\left(Y_{1}\right) \subset \sigma\left(Y_{2}\right) \subset \ldots \subset \sigma\left(Y_{j}\right) \subset \sigma\left(Y_{j+1}\right) \subset \ldots$, and there is no $n$ satisfying $Y_{n+1}=Y_{n+2}=\ldots$ Then $\sigma(X)=S$.

Proof. Both (s1) and (s2) are satisfied by $\sigma$ in view of Definition 2.

By Definition 2 and Lemma 1(4), we carry out $\sigma(\sigma(A))=\{y: \rho(\sigma(A) \cup y)=$ $\rho(\sigma(A))\}$, and $\rho(A)=\rho(\sigma(A))=\rho(\sigma(A) \cup y)=\rho(A \cup y)$ for $y \in \sigma(\sigma(A))$, and hence, $\sigma(A)=\sigma(\sigma(A))$. Namely, (s3) is satisfied by $\sigma$.

Next to prove the satisfactory of (s4).

Because $y \notin \sigma(A)$, it means $\rho(A \cup y) \neq \rho(A)$. By Definition 1, Lemma 1(2) and Lemma 1(3), it follows $\rho(A)<\infty$ and $\rho(A \cup y)<\infty$, and so, $\rho(A \cup y)=\rho(A)+1$. On the other hand, $y \in \sigma(A \cup x)$ implies $\rho(A \cup x \cup y)=\rho(A \cup x) \leq \rho(A)+1$. Hence, in view of Lemma 1 and the above, it brings about $\rho(A \cup y) \leq \rho(A \cup x \cup y) \leq$ $\rho(A)+1=\rho(A \cup y)$, and further, $\rho(A \cup x \cup y)=\rho(A \cup y)$. Thus, $x \in \sigma(A \cup y)$ holds.

Next, to prove (s5).

Assume $Y_{1} \notin \mathcal{J}$. This follows $Y_{1} \subseteq \sigma(\emptyset)$, a contradiction to $\sigma(\emptyset) \subset \sigma\left(Y_{1}\right) . Y_{2} \notin \mathcal{J}$ and $Y_{1} \subset Y_{2}$ carries out $\sigma\left(Y_{2}\right)=\sigma\left(Y_{1}\right)$, a contradiction. By the induction, we get $Y_{j} \in \mathcal{J}$ for any $j \in\{1,2, \ldots\}$.

By the supposition for $\left\{Y_{j}: j=1,2, \ldots\right\}$, we know $\left\{\left|Y_{j}\right|: j=1,2, \ldots\right\}=\{j$ : $j=1,2, \ldots\}$ and $Y_{j} \in \mathcal{J},(j=1,2, \ldots)$. So, it should have $|X|=\infty$.

If $X \in \mathcal{J}$. Then $\rho(X)=\infty$ is true. Obviously, $\sigma(X)=S$ holds.

If $X \notin \mathcal{J}$. Because $\rho(X)=\sup \{|Y|: Y \subset \subset X, Y \in \mathcal{J}\} \supseteq \sup \left\{\left|Y_{j}\right|: j=\right.$ $1,2, \ldots\}=\infty$ yields out $\rho(X)=\infty$. Thus, we yield out $\sigma(X)=\{y: \rho(X \cup y)=$ $\rho(X)=\infty\}=S$.

(s3) and Definition 2 together assure that $\sigma(A)$ is closed for $A \subseteq S$.

We can now prove the closure axiom systems of a pi-space.

Theorem 1(Closure axioms). A function $\sigma: 2^{S} \rightarrow 2^{S}$ is the closure operator of a pi-space $M$ on $S$ if and only if it satisfies (s1)-(s5).

Proof. Let $\sigma$ be the closure operator of $M$. Lemma 2 has shown that $\sigma$ satisfies (s1)-(s5).

Conversely, let $\sigma$ satisfy (s1)-(s5).

Define the collection $\mathcal{J}(\sigma)$ of subsets of $S$ by, for $A \subseteq S$ when $|A|<\infty . A \in \mathcal{J}(\sigma) \Longleftrightarrow x \in A \Rightarrow x \notin \sigma(A \backslash x)$. when $|A|=\infty$. $A \in \mathcal{J}(\sigma) \Longleftrightarrow$ $\forall B \subset A$ : if $|B|<\infty$, it follows $x \in B \Rightarrow x \notin \sigma(B \backslash x)$;

if $|B|=\infty$, it follows $B \in \mathcal{J}(\sigma)$ and $\sigma(B)=\sigma(A)=S$.

We show that $\mathcal{J}(\sigma)$ is the collection of independent subsets of a pi-space.

The null set belongs to $\mathcal{J}(\sigma)$ evidently. That is, (i1) is true.

If $A \in \mathcal{J}(\sigma)$. Suppose $B \subseteq A$ but $B \notin \mathcal{J}(\sigma)$.

Assume $|A|<\infty$. It causes that there is $x \in B$ satisfying $x \in \sigma(B \backslash x)$. Hence, by (s2), $x \in \sigma(A \backslash x)$ holds, and so, $A \notin \mathcal{J}(\sigma)$, a contradiction.

Assume $|A|=\infty$. It easily brings about the hold of (i2) by the above proof and the definition of $\mathcal{J}(\sigma)$. 
Next we prove the hold of (i3). We will fulfill this proof by two steps .

Let $A, B \subset \subset S$. If $A, B \in \mathcal{J}(\sigma),|A|=|B|+1$, and in addition, for any $x \in A \backslash B$, it has $B \cup x \notin \mathcal{J}(\sigma)$. Then, we call $(A, B)$ a pair of contrary.

Step 1. To prove that if $I \in \mathcal{J}(\sigma)$ but $I \cup x \notin \mathcal{J}(\sigma)$, then $x \in \sigma(I)$.

If $|I|=\infty$. Then by the definition of $\mathcal{J}(\sigma)$, it leads to $\sigma(I)=S$, and further, for $I \cup x \notin \mathcal{J}(\sigma)$, it assures to $I \cup x \subseteq S$, and so, $x \in \sigma(I)$.

If $|I|<\infty$. By the definition of $\mathcal{J}(\sigma), I \cup x \notin \mathcal{J}(\sigma)$ must cause that there is $y \in I \cup x$ satisfying $y \in \sigma(I \cup x \backslash y)$.

Suppose $y=x$. The need result is evidently true.

Suppose $y \neq x$. This makes $y \in I$. According to $I \in \mathcal{J}(\sigma)$, it shows $y \notin \sigma(I \backslash y)$. Recalling $y \in \sigma(I \cup x \backslash y)=\sigma((I \backslash y) \cup x)$ and (s4), we will earn $x \in \sigma((I \backslash y) \cup y)=$ $\sigma(I)$.

Step 2. To testify that $\mathcal{J}(\sigma)$ satisfies (i3).

Perhaps, for $A \subseteq S$, there are many $C \in \mathcal{J}(\sigma)$ such that $(A, C)$ is a pair of contrary. We notice $|A|<\infty$ is fixed. Hence, for $A$ and a pair of $(A, C)$ of contrary, $|A \cap C|<|A|$ is fixed. Thus, we can choose a pair of contrary $(A, B)$ such that $|A \cap B|$ is maximal.

Let $y \in A \backslash B$. In light of $A \in \mathcal{J}(\sigma)$ and the definition of $\mathcal{J}(\sigma)$, we obtain $y \notin \sigma(A \backslash y)$.

If $B \subseteq \sigma(A \backslash y)$. Then, by (s2) and (s3), it causes $\sigma(B) \subseteq \sigma(\sigma(A \backslash y))=\sigma(A \backslash y)$. Thus, $y \notin \sigma(B)$ is true according to $y \notin \sigma(A \backslash y)$. Furthermore, by Step 1, $B \cup y \in$ $\mathcal{J}(\sigma)$ holds. This follows a contradiction to $(A, B)$ as a pair of contrary. In other words, $B \nsubseteq \sigma(A \backslash y)$.

Let $z \in B \backslash \sigma(A \backslash y)$. In light of (s1) and $z \neq y$, it gets $z \in B \backslash A$. Combining $z \notin \sigma(A \backslash y), A \backslash y \subseteq A$ and (i2) with Step 1, it yields out $(A \backslash y) \cup z \in \mathcal{J}(\sigma)$. Set $D=(A \backslash y) \cup z$. We may easily obtain $|D|=|A|>|B|$ and $D \cap B=(A \cap B) \cup z$. So $|D \cap B|>|A \cap B|$ holds. But the maximality of $|A \cap B|$ compels $(D, B)$ not to be a pair of contrary. We may state that there is some $e \in D \backslash B$ such that $B \cup e \in \mathcal{J}(\sigma)$. We notice that $e \in D \backslash B=((A \backslash y) \cup z) \backslash B=(A \backslash y) \backslash B \subseteq A \backslash B$ and $B \cup e \in \mathcal{J}(\sigma)$ together produces a contradiction to $(A, B)$ as a pair of contrary.

Therefore, $\mathcal{J}(\sigma)$ satisfies (i3).

Summing up, by Definition 1, there is a pi-space $M$ on $S$ with $\mathcal{J}(\sigma)$ as its collection $\mathcal{J}$ of independent sets.

Finally, we testify that $\sigma$ and the closure operator $\sigma_{M}$ of $M$ coincide. We will finish our proof by $(S T 1)-(S T 5)$.

(ST1) To prove: for any $\overline{A \in \mathcal{J}}$ and $|A|<\infty$, it has $\sigma(A)=\{y: \rho(A \cup y)=\rho(A)\}$.

Suppose not. Because (s1) implies $A \subseteq \sigma(A)$, it exists $x \in \sigma(A) \backslash A$ satisfying $\rho(A \cup x) \neq \rho(A)$. In virtue of Lemma $1(2), \rho(A)=|A|<\infty$ and $\rho(A \cup x) \neq \rho(A)$, it obtains $\rho(A \cup x)=\rho(A)+1=|A|+1$, i.e. $A \cup x \in \mathcal{J}=\mathcal{J}(\sigma)$. By the definition of $\mathcal{J}(\sigma), x \notin \sigma((A \cup x) \backslash x)=\sigma(A)$ is true, a contradiction.

$(S T 2)$ To prove: for any $A \in \mathcal{J}(\sigma)$ and $|A|=\infty$, it has $\sigma(A)=\{y: \rho(A \cup y)=$ $\rho(A)\}=S$.

Because $A \in \mathcal{J}(\sigma)$ and $|A|=\infty$, by the definition of $\mathcal{J}(\sigma)$, it causes $\sigma(A)=S$, and by Definition 1 , it obtains $\rho(A)=\infty$. So, for any $y \in S$, in virtue of Lemma 1 , 
$\rho(A \cup y) \geq \rho(A)$ is correct, and hence, $\rho(A \cup y)=\infty$. Namely, $\rho(A \cup y)=\rho(A)=\infty$ and $\sigma_{M}(A)=S$. Moreover, $\sigma(A)=\sigma_{M}(A)=S$.

(ST3) Let $X \subseteq S$. To prove: if there is $I_{X} \subseteq X, I_{X} \in \mathcal{J}(\sigma)$ and $\left|I_{X}\right|=\infty$, then $\sigma(X)=\{y: \rho(X \cup y)=\rho(X)\}=S$.

According to Definition $1, \rho\left(I_{X}\right)=\rho(X)=\infty$ holds. In view of $(S T 2)$, it brings about $\sigma(X)=\sigma\left(I_{X}\right)=\{y: \rho(X \cup y)=\rho(X)\}=S$.

(ST4) Let $X \subseteq S$ and $X \notin \mathcal{J}(\sigma)$. To prove: if there is a positive integer $m$ such that for all $I \subseteq X$ and $I \in \mathcal{J}(\sigma),|I| \leq m$ is true, then $\sigma(X)=\{y: \rho(X \cup y)=\rho(X)\}$

Actually, we may equivalently suppose that there is $I_{X} \subseteq X$ and $I_{X} \in \mathcal{J}(\sigma)$ satisfying $\left|I_{X}\right|=m$, and besides, for any $I \subseteq X$ and $I \in \mathcal{J}(\sigma)$, it always gets $|I| \leq\left|I_{X}\right|$.

Under this event, it is not difficult to see $\rho(X)=m$.

For any $y \in X \backslash I_{X}$, by the selection of $I_{X}$, it gets $I_{X} \cup y \notin \mathcal{J}(\sigma)$. In light of Step 1 , we carry out $y \in \sigma\left(I_{X}\right)$. So $X=I_{X} \cup\left(X \backslash I_{X}\right) \subseteq \sigma\left(I_{X}\right)$ is true according to (s1). Further, by (s2) and (s3), it follows $\sigma(X) \subseteq \sigma\left(\sigma\left(I_{X}\right)\right)=\sigma\left(I_{X}\right) \subseteq \sigma(X)$, and hence, $\sigma(X)=\sigma\left(I_{X}\right)$.

By $(S T 1)$, it shows $\sigma\left(I_{X}\right)=\left\{y: \rho\left(I_{X} \cup y\right)=\rho\left(I_{X}\right)\right\}$. Besides, $I_{X} \cup y \notin \mathcal{J}(\sigma)$ for $y \in \overline{X \backslash I_{X}}$ also tells us $\rho(X \cup y)=\rho\left(I_{X} \cup y\right)=\rho\left(I_{X}\right)$. Thus,

$\sigma\left(I_{X}\right)=\left\{y: \rho\left(I_{X} \cup y\right)=\rho\left(I_{X}\right)=\rho(X)\right\}=\{y: \rho(X \cup y)=\rho(X)\}=\sigma(X)$.

$(S T 5)$ Let $X \subseteq S$ and $X \notin \mathcal{J}(\sigma)$. To prove: if $\left|I_{X}\right|<\infty$ is true for any $I_{X} \subseteq X$ and $\overline{I_{X} \in \mathcal{J}}(\sigma)$, and in addition, there is no positive integer $m$ satisfying $\left|I_{X}\right| \leq m$ for all $I_{X} \subseteq X$ and $I_{X} \in \mathcal{J}(\sigma)$, then $\sigma(X)=\{y: \rho(X \cup y)=\rho(X)\}$.

Under this supposition and considering with (i2), we may indicate that for any positive integer $n$, it can always find out $Y \in \mathcal{J}(\sigma)$ and $Y \subset X$ satisfying $|Y|=n$. Additionally, for any $I \in \mathcal{J}(\sigma)$ and $I \subset X$, it always has $|I|<\infty$.

By Definition 1, this means $\rho(X)=\infty$. So, for any $y \in S, \rho(X \cup y) \geq \rho(X)=\infty$ holds by Lemma 1(1), and further, $\rho(X \cup y)=\infty=\rho(X)$. That is, $\{y: \rho(X \cup y)=$ $\rho(X)\}=S$.

On the other hand, for $\left|I_{1}\right|=1$ and $X \supseteq I_{1} \in \mathcal{J}(\sigma)$, by the given supposition and (i3), we will find out a series of $Y_{j} \in \mathcal{J}(\sigma)$ such that $Y_{1}=I_{1}, Y_{1} \subset Y_{2} \subset$ $Y_{3} \subset \ldots \subset Y_{j} \subset Y_{j+1} \subset \ldots \subset X,\left|Y_{j}\right|=j,(j=1,2, \ldots)$, and there is no positive integer $n$ compelling to all of $Y_{j}$ satisfying $\left|Y_{j}\right| \leq n,(j=1,2, \ldots)$. Additionally, by the definition of $\mathcal{J}(\sigma)$, we also obtain $\sigma(\emptyset) \subset \sigma\left(Y_{1}\right) \subset \sigma\left(Y_{2}\right) \subset \ldots \subset \sigma\left(Y_{j}\right) \subset$ $\sigma\left(Y_{j+1}\right) \subset \ldots$ In virtue of the supposition, it does not have any $n$ satisfying $Y_{n+1}=Y_{n+2}=\ldots$. Hence, by (s5), it has $\sigma(X)=S$.

Therefore, $\sigma(X)=\{y: \rho(X \cup y)=\rho(X)\}$ is carried out.

Some descriptions in the proof of Theorem 1 are a little similar to that for finite matroids (cf. [18]). Considered the discussion for the closure axioms of a finite matroid in [18, pp. 28-30\&20, p.19], we find out that it uses the idea of bases of a finite matroid to fulfil their proofs. But, in this article, we do not use the idea of bases of a pi-space, because by $[17,20]$, we know that a pi-space needs not to have bases at all. Thus, our proof of closure axioms for a pi-space is quite different from that of finite matroids. 
Now we look at the collection of closed sets of a pi-space. Since the closure operator $\sigma$ of a pi-space satisfies (s1)-(s5), we have the following basic theorem of lattice theory.

Theorem 2. The closed sets of a pi-space form a complete semi-modular lattice under set inclusion, and each of its elements is join of atoms.

Proof. Routine verification.

$[20,21]$ point out the notable position of geometric lattices in finite matroid theory. How about the position of geometric lattice in pi-space theory?

The definition of geometric lattice in $[2$, p. 80$]$ is a little different from $[3$, p.234, Definition 1]. The reason is that [3, p.234, Definition 1] does not ask a geometric lattice to be a finite length but $[2$, p.80] does. We will give an instance to show that this brings some different result for pi-spaces. The instance is given as: Let $S=\{1,2, \ldots\}$ and $\mathcal{J}(S)=\{X$ : there is $x \in S$ satisfying $X \subseteq S \backslash x\}$. Obviously, $(S, \mathcal{J}(S))$ is a pi-space with $\sigma_{S}$ as its closure operator. In addition, for any $X \subset \subset S$, it gets $\sigma_{S}(X)=X$ and $\sigma_{S}(Y)=S$ for any $Y \subseteq S$ and $|Y|=\infty$. Hence, the length of $\sigma_{S}(Y)$ is $\sup \{|X|: X \subset \subset Y\}=\infty$. The definition of [2, p.80] relative to geometric lattice will not allow $L(S)$, the lattice of all closed sets of $(S, \mathcal{J}(S))$ under set inclusion, to be geometric because it has not finite length. But [3, p.234, Definition 1] allows it to be geometric.

[7] presents a way for a matroid of arbitrary cardinality to have its closed sets corresponding to a finite length geometric lattice. According to the results in [7] and the relation between pi-spaces and matroids of arbitrary cardinality, we conclude that there is not a geometric lattice corresponding to the poset which is the set of all of the closed sets of a pi-space by set inclusion (Here the definition of geometric is come from $[2$, p. 80]).

$[20$, p.388] informs us that in general, we no longer have a geometric lattice for an independence space since for infinite cases, a geometric lattice is defined to have only finite dimension (i.e. finite height). Thus, generally, we no longer have a geometric lattice for a pi-space (The geometric definition here is adopted with $[2$, $3])$.

[20, p. 388] presents the closure axioms of an independence spaces. Considered Theorem 1 with [20, p.388], we may easily indicate that if $\sigma$ is the closure operator of an independence space $M$, then it is the closure operator of the pi-space $M$. This also states that an independence space is a pi-space. This statement is the same to the result yielded out of the definitions of independence space and pi-space provided in $[20]$.

The following two sections will apply Theorem 1 to discuss some properties of pi-spaces.

\section{Dual}

We know that the orthogonality of a finite matroid $M_{f}$ is its dual of $M_{f}$, and 
vice versa. In the following, we will provide an example to demonstrate that there is no dual function on the collection of pi-spaces on an infinite set $S$. In other words, there is no dual operation among the set of pi-spaces defined on the same infinite background.

Let $M=(S, \mathcal{J})$ be a pi-space with $\sigma$ as its closure operator. Similarly to [18, 20, 21], we earn the "dual" of $\sigma$ is defined as $\sigma^{*}(X)=X \cup\{x: x \notin \sigma(S \backslash(X \cup x))\}$.

Example. Let $S=\{1,2,3, \ldots\}$ and $k=4$. If $\mathcal{J}_{k}=\{X \subseteq S:|X| \leq k\}$. Then $\mathcal{J}_{k}$ is obviously the set of independent sets of a pi-space $M$ on $S$. Let $\sigma$ be its closure operator. By the definition of the closure operator, we obtain $\sigma(X)=X$ when $X \subseteq S$ and $|X|<k ; \sigma(X)=S$ when $X \subseteq S$ and $|X| \geq k$.

According to the definition of the above $\sigma^{*}$, we earn $\sigma^{*}(Y)=S$ when $|S \backslash Y| \leq k$; $\sigma^{*}(Y)=Y$ when $|S \backslash Y| \geq k+1$.

Let $f=\sigma^{*}$. Then $f^{*}(X)=X \cup\{x: x \notin f(S \backslash(X \cup x))\}=X \cup\{x: x \notin$ $\left.\sigma^{*}(S \backslash(X \cup x))\right\}$.

Set $X_{0}=\{1,2\}$. Then $\sigma^{*}\left(S \backslash\left(X_{0} \cup x\right)\right)=S \backslash\left(X_{0} \cup x\right)$ is true for any $x \notin X_{0}$, and so, $x \notin \sigma^{*}\left(S \backslash\left(X_{0} \cup x\right)\right)$ is led to. That is to say, $\left(\sigma^{*}\right)^{*}\left(X_{0}\right)=f^{*}\left(X_{0}\right)=$ $X_{0} \cup\left(S \backslash X_{0}\right)=S$. But, at the same time, we obtains $\sigma\left(X_{0}\right)=\{1,2\}$. Namely, $\sigma \neq\left(\sigma^{*}\right)^{*}$.

$[18,20,21]$ represent that there is the dual function on the collection of finite matroids on a finite set $E$. Additionally, [18, 20, 21] and Definition 1 show us that every finite matroid on $E$ is a pi-space on $E$. But the above Example states that there is no dual for some pi-space. Combining the beyond, we produce the following result.

Theorem 3. There is no dual function on the collection of pi-spaces on an infinite set $S$.

\section{Automorphism group}

Welsh in [20] shows us that one of important parts in finite matroid theory is the automorphism groups of finite matroids. It is also a relationship between group theory and finite matroids. Using this relationship, group theory is applied in the research of finite matroid theory, and also vice versa. Even though, up till now, it still exists some open problems in this field (cf. [20, p. 331]).

We close this paper with an account of the work on the group of automorphism of a pi-space. As finite matroids, we define the automorphism of a pi-space as follows.

Definition 3. Let $M$ be a pi-space on $S$. A bijection $\pi: S \rightarrow S$ is an automorphism of $M$ if $\pi X$ is independent in $M$ if and only if $X$ is independent in $M$.

The automorphism group of $M$ is the collection of automorphisms of $M$ with the obvious operation of composition. It will be denoted by $A(M)$. 
[16] discusses "closure axioms" for the automorphism group of a finite matroid $M_{f}=\left(E, \mathcal{J}_{f}\right)$. Here, the "closure axioms" means that if $\pi$ is a permutation on $E$, then $\pi \in A\left(M_{f}\right)$ if and only if " $X \in \mathcal{F}_{f} \Leftrightarrow \pi X \in \mathcal{F}_{f}$ ", where $\mathcal{F}_{f}$ is the collection of closed sets of $M_{f}$. Thus, for pi-spaces, we have the following properties.

Theorem 4. Let $M=(S, \mathcal{J})$ be a pi-space with $\mathcal{F}, \sigma$ as its collection of closed sets and its closure operator respectively. Then there are the following statements.

(1) Let $\pi \in A(M)$ and $X \subseteq S$. It induces $X \in \mathcal{F} \Leftrightarrow \pi X \in \mathcal{F}$.

(2) Define $\pi: S \rightarrow S$ a bijection satisfying $A \in \mathcal{F} \Leftrightarrow \pi A \in \mathcal{F}$ as the closed automorphism of $M$. Then $\mathcal{F} A(M)$ the collection of closed automorphisms of $M$ with the obvious operation of composition is a group.

(3) $A(M) \subseteq \mathcal{F} A(M)$.

Proof. First of all, we prove that for any $X \subseteq S$, the equality $\rho(X)=\rho(\pi X)$ is right for any $\pi \in A(M)$.

Because $\rho(X)=\sup \{|Y|: Y \subset \subset X, Y \in \mathcal{J}\}, \rho(\pi X)=\sup \{|Z|: Z \subset \subset \pi X, Z \in$ $\mathcal{J}\}$ and $\pi$ is a bijection, it brings about that $|\pi Y|=|Y|$ holds for any $Y \subset \subset X$ and $Y \in \mathcal{J}$. In addition, for any $Z \subset \subset \pi X$ and $Z \in \mathcal{J}$, there is $Y_{Z} \subset \subset X$ and $Y_{Z} \in \mathcal{J}$ satisfying $Z=\pi Y_{Z}$, and further, $|Z|=\left|\pi Y_{Z}\right|=\left|Y_{Z}\right|$. Thus, it follows $\rho(\pi X) \leq$ $\rho(X)$. Similarly, owing to $\pi^{-1} \in A(M)$, it also brings about $\rho\left(\pi^{-1}(\pi X)\right) \leq \rho(\pi X)$, i.e. $\rho(X) \leq \rho(\pi X)$. Therefore, we obtain $\rho(\pi X)=\rho(X)$.

Secondly, we prove (1) as follows.

Let $X \in \mathcal{F}$. Since $X \in \mathcal{F} \Leftrightarrow X=\sigma(X) \in \mathcal{F} \Leftrightarrow X=\{y: \rho(X \cup y)=\rho(X)\} \in \mathcal{F}$. By $\pi \in A(M)$, it yields out $\pi X=\pi \sigma(X)=\{\pi y: \rho(X \cup y)=\rho(X)\}=\{\pi y$ : $\rho(\pi(X \cup y))=\sigma(\pi X)\}=\{\pi y: \rho(\pi X \cup \pi y)=\rho(\pi X)\}=\{\pi y: \rho(\pi X \cup \pi y)=$ $\rho(X)\}=\{\pi y: \rho(X \cup y)=\rho(\pi X \cup \pi y)=\rho(X)\}$, and in addition, $\sigma(\pi X)=\{t:$ $\rho(\pi X \cup t)=\rho(\pi X)\}=\{\pi s=t: \rho(\pi X \cup \pi s)=\rho(X)\}=\{\pi s: \rho(\pi(X \cup s))=$ $\rho(X)\}=\{\pi s: \rho(X \cup s)=\pi(X)\}$. This follows $\pi X=\sigma(\pi X)$. So $\pi X \in \mathcal{F}$ is found out.

Since $\pi \in A(M) \Rightarrow \pi^{-1} \in A(M)$. By the above, it is easy to obtain $\pi X \in \mathcal{F} \Leftrightarrow$ $X \in \mathcal{F}$.

Thirdly, (2) is straightforward.

Fourthly, according to the definitions of $A(M)$ and $\mathcal{F} A(M)$, and at the same time, considering with (1), (3) is born.

We notice that Welsh provides a problem in $[20$, p. 331, Exercise 4]. According to the description in [20, p. 1, Line 8], [20, p. 331, Exercise 4] is a real open problem. The problem is "Is it true that for any group $H$ there is a matroid design with automorphism group isomorphic to $H$ ?". We know that for a finite matroid, its automorphism group is finite. Hence

(I) When $H$ is finite, this problem is solved by [20, p. 330, Theorem 2].

(II) When $H$ is infinite, there will not have any finite matroid design with automorphism group isomorphic to $H$. Since pi-spaces are the generalization of finite matroids. So, in general, when $H$ is infinite, we guess that there is a matroid design a pi-space with automorphism group isomorphic to $H$. This guess will be checked on some day. 
Acknowledgements The authors thank National Nature Science Foundation of China for grant (No. 60974082) and also thank Science and Technology Item of Baoding, China for grant (No. 11ZG005)

\section{References}

[1] D. Betten and W. Wenzel, On linear spaces and matroids of arbitrary cardinality, Algebra Universalis, 49(2003), 259-288.

[2] G. Birkhoff, Lattice Theory, 3rd. ed. Amer. Math. Society, Providence, 1967.

[3] G. Grätzer, General Lattice Theory, 2nd. ed. Birkhäuser Verlag, Basel, 1998.

[4] T. W. Hungerford, Algebra, Springer-Verlag, New York, 1974.

[5] F. Maeda and S. Maeda, Theory of Symmetric Lattices, Springer-Verlag, Berlin, 1970.

[6] H. Mao and S. Liu, Remarks on external elements in independence spaces, Southeast Asian Bulletin of Mathematics, 29(2005), 939-944.

[7] H. Mao, On geometric lattices and matroids of arbitrary cardinality, Ars Combinatoria, 8(2006), 23-32.

[8] H. Mao, An axiom scheme for cyclic flats of a matroid of arbitrary cardinality, International J. of Pure and Appl. Math., 41(8)(2007), 1107-1122.

[9] H. Mao, Characterization of disconnected matroids, Algebra Colloquium, 15(1)(2008), 129-134.

[10] H. Mao, Single element extensions of matroids of arbitrary cardinality, Acta Mathematica Sinica (Chinese series), 50(6)(2007), 1271-1280(in Chinese).

[11] H. Mao, Independence spaces generated by a graph, East-West J. of Math., 9(1)(2007), 63-68.

[12] H. Mao and G. Wang, Some properties of base-matroids of arbitrary cardinality, Rocky Mountain J. of Math., 409(1)(2010), 291-303.

[13] H. Mao, The sub-independence-space, Chinese Quart. J. of Math., 25(2)(2010), 293299.

[14] H. Mao, Paving matroids of arbitrary cardinality, Ars Combinatoria, 90(2009), 245256.

[15] H. Mao and G. Wang, On the basis graph of a matroid of arbitrary cardinality, Advances in Pure and Appl. Math., (2)(2010)275-284.

[16] H. Mao and S. Liu, Relations between some axiom systems for matroids and the automorphism groups of a matroid, J. of Xidian University, 28(1)(2001), 48-51(in Chinese)

[17] J. Oxley, Infinite Matroids, in Matroid Application, ed. by Neil White, Cambridge University Press, Cambridge, 1992, 73-90.

[18] J. Oxley, Matroid Theory, Oxford University Press, New York, 1992. 
[19] M. Stern, Semimodular Lattices: Theory and Applications, Cambridge University Press, Cambridge, 1999.

[20] D. J. A. Welsh, Matroid Theory, Academic Press, London, 1976.

[21] N. White, Theory of Matroids, Cambridge University Press, Cambridge, 1976. 